\title{
NORMAS PARA PUBLICACIÓN
}

La Revista Científica Odontológica de la Escuela de Estomatología de la UCSUR, tiene las siguientes secciones: Artículos originales, artículos de revisión, reporte de caso, contribución clínica- docente, entrevistas al experto, artículos de opinión. Para la publicación de alguna de las secciones se debe presentar al Comité Editorial una copia impresa y formato electrónico de la redacción, el texto en Microsoft Word y las tablas en formato Excel, a continuación se describen las normas:

\section{Descripción de la presentación:}

- Tipo de letra Times New Roman, número 12 a espacio simple en papel blanco ISO A4.

- Texto escrito en una sola cara.

- Márgenes de $25 \mathrm{~mm}$ interno y externo y $30 \mathrm{~mm}$ superior e inferior.

- Numeración en el ángulo superior derecho de cada página.

Formato para artículos originales: Deben tener las siguientes partes: Título, autores, resumen en español e inglés, palabras clave, texto, referencias, tablas, figuras con leyendas y agradecimientos. Cada uno de estos componentes debe aparecer en una página nueva.

Título: Conciso e informativo (máximo 50 caracteres incluidos los espacios).

Autores: Apellido paterno, primer nombre e inicial del segundo nombre, indicar el grado académico, institución a la que pertenecen, nombre completo, dirección, teléfono y correo electrónico del autor principal.

Resúmenes y palabras clave: Los resúmenes deben presentarse en español e inglés y no deben contener más de 250 palabras. Las palabras clave deben ser máximo cuatro y deben ser de uso corriente en la literatura científica como los utilizados en el DeCS, Mesh, Index Medicus y Dental.

Texto: Los trabajos originales se presentarán divididos en las siguientes secciones: Introducción, materiales y métodos, resultados, discusión y conclusiones.

Agradecimientos: Especificarlos en forma sucinta. (No es necesario)

Referencias bibliográficas. Enumeradas consecutivamente en orden de aparición en el texto, señalados con números arábicos en superíndice colocados después de la puntuación. Los títulos de revista deben ser abreviados de acuerdo al estilo utilizado en el Index Medicus. Referir a todos los autores, cuando sean seis o menos, en el caso de haber siete o más autores referir sólo los tres primeros y agregar las palabras y cols. Utilizar las Normas de Vancouver.

Tablas: Presentar las tablas a doble espacio en una hoja aparte en formato Excel. Enumerar las tablas consecutivamente en orden de aparición en el texto, con un breve título para cada una de ellas en la parte superior.

Figuras: Las fotografías se presentarán en hoja aparte, numeradas en forma consecutiva y con un breve título parte inferior para cada una de ellas. Se presentarán también los archivos originales, en formato TIFF o JPEG.

Los artículos propuestos para su publicación en la revista serán remitidos adjuntando el curriculum vitae resumido del autor, con teléfonos, correo electrónico y dirección postal. El Comité Editor asignará el texto propuesto a uno o dos árbitros externos o internos para su evaluación. Estos árbitros remitirán su opinión dentro de los 30 días calendario siguientes. De haber controversia, el Comité Editor tendrá facultad dirimente en un plazo de 15 días más.

Los reportes de caso: Deben adjuntarse al formato de un artículo original, teniendo en cuenta que el sólo varía el texto en: Introducción, reporte de caso, discusión y conclusiones.

Los artículos de revisión: Constarán de una presentación concisa de la literatura más reciente sobre el tema que se revisa, deben adjuntarse al formato de un artículo original, teniendo en cuenta que el sólo varía el texto en: Introducción, principales aportes del tema (como subtítulos) y conclusiones.

Contribución clínica - docente y entrevista al experto o artículos de opinión: Esta sección busca publicar temas que reflejen opiniones y alcances sobre temas clínicos y docentes que estimulen la discusión científica y contribuyan al conocimiento del área. La presentación de estas secciones incluye la descripción del texto y la incorporación de fotografías de los involucrados. 